\title{
Fission barriers for Po nuclei produced in complete fusion reactions with heavy ions
}

\author{
R. N. Sagaidak \\ Flerov Laboratory of Nuclear Reactions, Joint Institute for Nuclear Research, RU-141980, Dubna, Moscow region, Russia
}

A. N. Andreyev

Instituut voor Kern-en Stralingsfysica, University of Leuven, Celestijnenlaan 200 D, B-3001 Leuven, Belgium

(Received 6 October 2008; published 22 May 2009)

\begin{abstract}
Evaporation residues and fission excitation functions obtained in complete fusion reactions leading to Po compound nuclei have been analyzed in the framework of the standard statistical model. Macroscopic fission barriers deduced from the cross-section data analysis are compared with the predictions of various theoretical models and available data. A drop in the Po barriers with the decrease in a neutron number was found, which is stronger than predicted by any theory. The presence of entrance channel effects and collective excitations in the compound nucleus decay is considered as a possible reason for the barrier reduction.
\end{abstract}

DOI: 10.1103/PhysRevC.79.054613

PACS number(s): 25.70.Jj, 24.60.Dr, 25.70.Gh, 27.80.+w

\section{INTRODUCTION}

According to the prediction of various theoretical and empirical models [1-4], Po nuclei might have the macroscopic (liquid drop) component of fission barriers ranging from $B_{f}^{m}=10-12 \mathrm{MeV}$ at $N=126$ to $B_{f}^{m} \lesssim 9 \mathrm{MeV}$ at $N \leqslant 110$. The ground-state microscopic component (shell correction energy) spans the range from $\Delta W_{\mathrm{gs}}=-12 \mathrm{MeV}$ to $1.5 \mathrm{MeV}$ for the same nuclei, according to the finite-range model calculations [5]. Bearing in mind that both components make up the height of the fission barrier $B_{f}=B_{f}^{m}-\Delta W_{\mathrm{gs}}$ (neglecting a small shell correction at the saddle point), Po nuclei offer a unique possibility to study the survival probability of both spherical and deformed nuclei at different excitation energies. According to the standard statistical model (SSM) approximations (see, e.g., Refs. [6,7]), the survivability of heavy fissile nuclei produced in fusion-evaporation reactions strongly depends on both components of fission barriers for nuclei in the de-excitation cascade of a compound nucleus (CN) formed in complete fusion; namely the macroscopic part reduces with the increase in an angular momentum $L$, whereas the shell effect fades out in the nuclear level density with the increase in an excitation energy.

Earlier, the analysis of the measured cross sections for evaporation residues (ER) around ${ }^{216} \mathrm{Th}$ produced in fusion reactions with ${ }^{40} \mathrm{Ar}$ [7] and heavier projectiles [8] showed no noticeable enhancement due to the shell stabilization around $N=$ 126 , despite an appreciable shell energy $\Delta W_{\mathrm{gs}}=-4.7 \mathrm{MeV}$ [5]. This result is in a sharp contrast to expectations based on the SSM calculations using intrinsic level densities [6]. The lack of stabilization against fission around $N=126$ nuclei was explained by the influence of collective excitations in terms of a reduced collective contribution to the level density in spherical nuclei [9]. In the later analysis of the production cross sections for nuclei obtained in the U projectile fragmentation, the expected stabilization against fission for spherical nuclei near $N=126$ was not found again [10]. However, with the inclusion of collective enhancement in the level density (CELD) the experimental data were well described. It is implied that CELD is essentially the same at the saddle point deformation (fission channel) for heavy nuclei, but it differs strongly in the ground-state (equilibrium) deformation of an evaporation channel. For well-deformed nuclei the collective enhancement factors above the saddle point and above the ground state are almost equal and correspond to rotational excitations in both configurations. In the case of spherical nuclei, the vibrational enhancement factor in the ground state is much smaller than the rotational one in the deformed saddle-point configuration. The lack of shell stabilization against fission observed for the $N=126$ shell was expected to have severe consequences for the production of spherical superheavy nuclei around the next closed neutron shell at $N=184$ [10].

In the mid-1980s, significant limitations for fusion in heavy systems were revealed in studies of fission-like processes proceeding in reactions with massive nuclei [11-13]. These limitations are caused by quasifission $(\mathrm{QF})$, the reseparation of two fissionlike fragments in a dinuclear system, which outwardly resembles $\mathrm{CN}$ fission but proceeds without the $\mathrm{CN}$ formation. Note that the QF effect reducing a complete fusion cross section was observed in rather asymmetric ${ }^{32} \mathrm{~S}+$ ${ }^{182} \mathrm{~W}$ combination leading to the ${ }^{214} \mathrm{Th}^{*} \mathrm{CN}$ [14]. Afterward quasifission was found in fission studies of reactions leading to less-fissile compound nuclei, e.g., QF was pronouncedly observed in ${ }^{48} \mathrm{Ca}$-induced reactions leading to $\mathrm{Ra}$ compound nuclei [15]. Recently, it was revealed in reactions with deformed target nuclei leading to the ${ }^{202} \mathrm{~Pb}^{*}$ [16] and ${ }^{202} \mathrm{Po}^{*}$ [17] compound nuclei. Rather unexpectedly the fusion suppression effect was observed in quite asymmetric combinations with the ${ }^{19} \mathrm{~F}$ projectile leading to the ${ }^{216} \mathrm{Ra}^{*} \mathrm{CN}$ in studies of the ER production. It followed from the comparison of ER cross sections with those obtained in the very asymmetric ${ }^{12} \mathrm{C}+{ }^{204} \mathrm{~Pb}$ reaction leading to the same $\mathrm{CN}$ [18]. Note that according to the fission studies [12,13], QF appears in reactions with $\mathrm{Mg}$ and heavier projectiles.

In recent work [19], the cross sections for the neutrondeficient ${ }^{186-192} \mathrm{Po}$ nuclei produced in fusion-evaporation reactions with the ${ }^{46} \mathrm{Ti}$ and ${ }^{50,52} \mathrm{Cr}$ ions have been analyzed. It has been shown that a very low macroscopic component corresponding to the scaling factor as low as $k_{f}=0.67$ 
at the liquid-drop (LD) fission barriers in the expression $B_{f}(L)=k_{f} B_{f}^{\mathrm{LD}}(L)-\Delta W_{\mathrm{gs}}$ should be used to reproduce very low production cross sections observed in the reactions. This work stimulates the analysis of the Po nuclei production in a wider region, from spherical nuclei around $N=126$ to well-deformed ones at $102 \leqslant N<110$ (observed in Ref. [19]). As mentioned above, at least two independent effects might cause the low production cross sections. (1) The CELD effect, which is significant for the spherical nuclei production. It might imitate the relatively low macroscopic component of fission barriers, if low production cross sections are observed in experiments and if the CELD effect is not taken into account in the framework of the SSM analysis. (2) The QF effect being solely the entrance-channel effect, which seems to be essential for reactions with massive and deformed nuclei. It does not depend on decay properties and deformations of nuclei formed in $\mathrm{CN}$ reactions.

In the present work we analyze the production of Po nuclei in the range of neutron numbers $104 \leqslant N \leqslant 126$. Available data on ER and fission cross sections obtained in different $\mathrm{CN}$ reactions are compared with calculations using the SSM approximations [6]. In our analysis experimental ER and fission cross sections are fitted with the adjustment of only the scaling factor $k_{f}$ at the LD fission barriers as mentioned above. As noted in Ref. [19], over the past two decades this approach has been extensively and successfully used by many authors and thorough discussion of its advantages and drawbacks can be found in the references of the article. Note that at present the SSM analysis of fusion cross-section data is one of the ways to obtain information on the macroscopic component of fission barriers for neutron-deficient heavy nuclei. We remind that the values derived with such analysis cannot be considered as the "experimental barriers" because their derivation is conditioned by some approximations of SSM. At the same time, these estimates can fill up missing information on this macroscopic characteristic of heavy nuclei and its isotopic properties.

In the next section (Sec. II) we review the model used for the analysis of ER and fission excitation functions. The available excitation functions measured in various complete fusion reactions are considered in Sec. III. The results of their analysis are presented and discussed in Sec. IV. The work is summarized in Sec. V.

\section{METHOD OF ANALYSIS}

The analysis is done within the standard approach, which treats the $\mathrm{CN}$ production (fusion) and de-excitation steps independently. The fusion part is described within the barrierpassing (BP) model, while the $\mathrm{CN}$ de-excitation is treated within the SSM. Both of them are implemented within the HIVAP code [6].

A conventional BP model that generally reproduces an experimental capture cross section as the barrier-passing one is used for the data analysis. The calculated BP cross section can be associated with the fusion cross section, implying that the fusion probability $P_{\text {fus }}=1$. The effect of coupling the entrance channel to other reaction channels is taken into account phenomenologically via the fluctuations of the radius-parameter $r_{0}$. The radius fluctuations are generated with a Gaussian distribution around its average $r_{0}=1.12 \mathrm{fm}$ with the barrier fluctuation parameter $\sigma\left(r_{0}\right)$ [20]. These fluctuations simulate coupling effects [21] at energies around the Coulomb barrier to a degree sufficient for the purpose of the present analysis. All the BP model parameters are fixed with the exception of the strength $V_{0}$ and the relative barrier fluctuation parameter $\sigma\left(r_{0}\right) / r_{0}$ in the nuclear exponential potential [20]. Variations of these parameters allow one to reproduce the experimental cross-section data for fission and ER production at subbarrier energies in calculations. Transmission coefficients through the potential barrier are calculated using the WKB approximation. Note that for strongly fissile compound nuclei the ER cross sections at energies well above the nominal fusion barrier [22] are weakly sensitive to the form of the nuclear potential and are mainly determined by SSM parameters.

In the SSM only intrinsic level densities are considered. The Reisdorf's expression [6] is used to calculate the macroscopic level-density parameters $\tilde{a}_{f}$ and $\tilde{a}_{v}$ in fission and evaporation channels, respectively. The scaling factor $k_{f}$ at the rotating LD fission barriers $B_{f}^{\mathrm{LD}}(L)$ [1] is used as the only fitting parameter in the expression $B_{f}(L)=k_{f} B_{f}^{\mathrm{LD}}(L)-\Delta W_{\mathrm{gs}}$, as it was done in previous cases (see Ref. [19] and references therein). Empirical masses [23] are used to calculate shell corrections $\Delta W_{\mathrm{gs}}$ (as a difference between empirical and LD masses [24]) as well as for the calculations of excitation and separation energies. The influence of the shell effects on the level density is included by an energy backshift in the level-density parameter that is exponentially damped with increasing excitation energy. The same damping constant $E_{D}=18.5 \mathrm{MeV}$ was used as in a number of the previous works [6-8, 19,25-28].

Usually, in the analysis of the most asymmetric combinations, excitation functions at high energies can be fitted with the variation of $k_{f}$ mainly. The lack of any fusion suppression $\left(P_{\text {fus }}=1\right)$ is evident if ER and fission excitation functions are fitted with the same parameter values of the nuclear potential and $k_{f}$ in the whole range of excitation energies. In less asymmetric combinations the absence of the QF (fusion suppression) effect is not evident (see Sec. I). The fusion probability value $\left(P_{\text {fus }}<1\right)$ might be phenomenologically introduced to describe ER excitation functions using the same scaling for fission barriers and other parameters of SSM, as obtained in the analysis of the most asymmetric combination leading to the same CN $[25,26]$.

In the present study we start our analysis with the very asymmetric ${ }^{16,18} \mathrm{O}+{ }^{186,188,190,192} \mathrm{Os}$ combinations leading to the ${ }^{202-210} \mathrm{Po}^{*}$ compound nuclei. Nuclei with $A_{\mathrm{CN}} \leqslant 202$ are produced in less asymmetric or in (nearly) symmetric combinations. Fusion suppression in the latter cases can be revealed by attempting to describe data with the $k_{f}$ value obtained in the analysis of asymmetric reactions leading to the same or a neighboring (more neutron-rich) $\mathrm{CN}$.

\section{CROSS-SECTION DATA ANALYSIS}

\section{A. Very asymmetric ${ }^{16,18} \mathrm{O}+$ Os reactions}

Fission excitation functions for Po compound nuclei were initially obtained in different combinations of the ${ }^{16,18} \mathrm{O}$ 


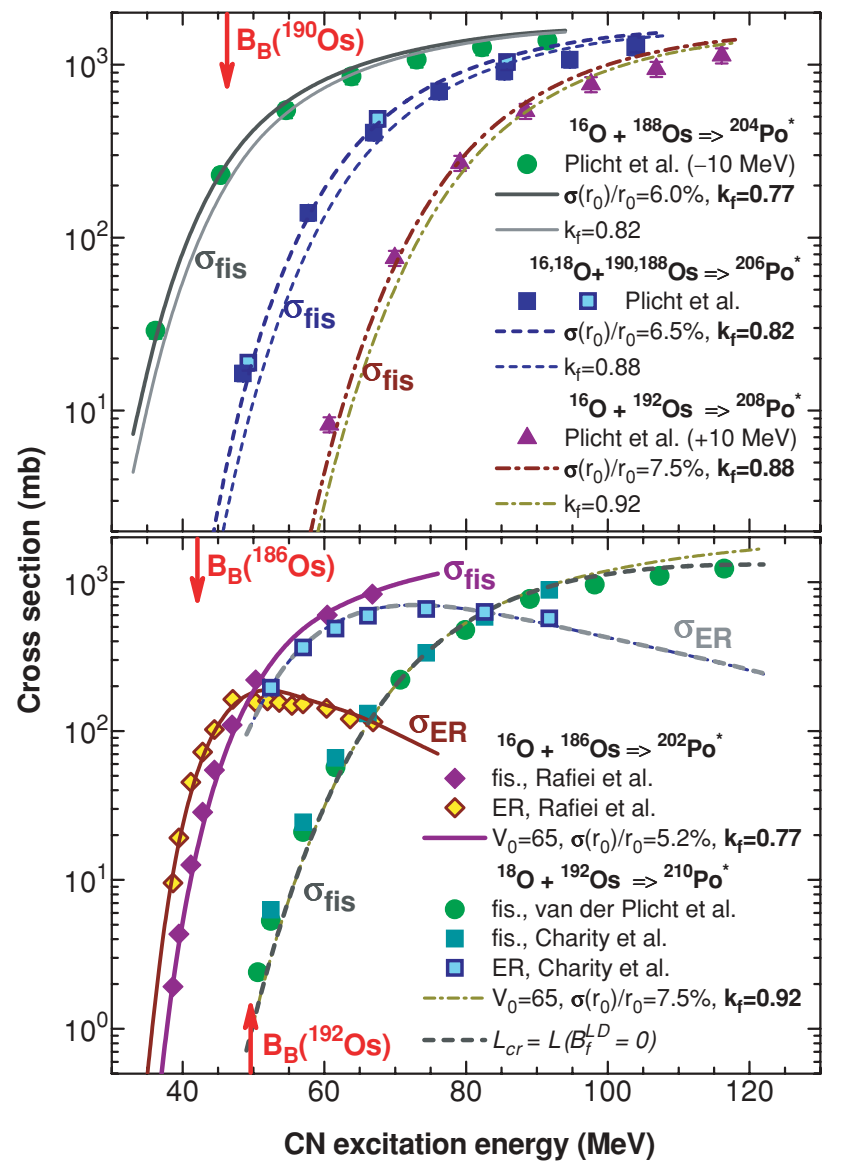

FIG. 1. (Color online) Fission and ER excitation functions obtained in the ${ }^{16,18} \mathrm{O}+{ }^{186,188,190,192} \mathrm{Os}$ reactions [29-31] (symbols) are shown together with our results of the SSM data analysis (lines). Calculations in upper panel correspond to the $L_{\mathrm{cr}}=L\left(B_{f}^{\mathrm{LD}}=0\right)$ restriction. Arrows with corresponding labels $B_{B}$ indicate positions of the nominal fusion barrier [22].

projectiles with the isotopes of ${ }^{188,190,192}$ Os [29]. These data together with the ER and fission cross sections measured later in the ${ }^{18} \mathrm{O}+{ }^{192} \mathrm{Os}$ reaction [30], as well as similar data obtained recently in ${ }^{16} \mathrm{O}+{ }^{186} \mathrm{Os}$ [31], are the starting point of the analysis.

In Fig. 1 fission and ER excitation functions obtained in Refs. [29-31] are shown together with our results of the SSM data analysis. Going from the most neutron-rich ${ }^{210} \mathrm{Po}^{*} \mathrm{CN}$ data to the most neutron-deficient ${ }^{202} \mathrm{Po}^{*}$ ones, the scaling parameter at the LD fission barriers has to be decreased from $k_{f}=0.92$ to 0.77 to describe the excitation functions in the whole region of the $\mathrm{CN}$ excitation energy (bottom panel). The analysis of fission data for the intermediate $\mathrm{CN}$ masses $A_{\mathrm{CN}}=208,206,204$ gives us the intermediate $k_{f}$ values (upper panel). The rotating LD model restriction for the critical angular momentum $L_{\mathrm{cr}}=L\left(B_{f}^{\mathrm{LD}}=0\right)$ corresponding to the disappearance of the $\mathrm{LD}$ component at high angular momenta allows one to better reproduce fission cross sections at high excitation energies (bottom panel). All the data are well fitted with the same value of the strength parameter $V_{0}=65 \mathrm{MeV} / \mathrm{fm}$ of the nuclear potential, whereas the fusion barrier fluctuation parameter is different

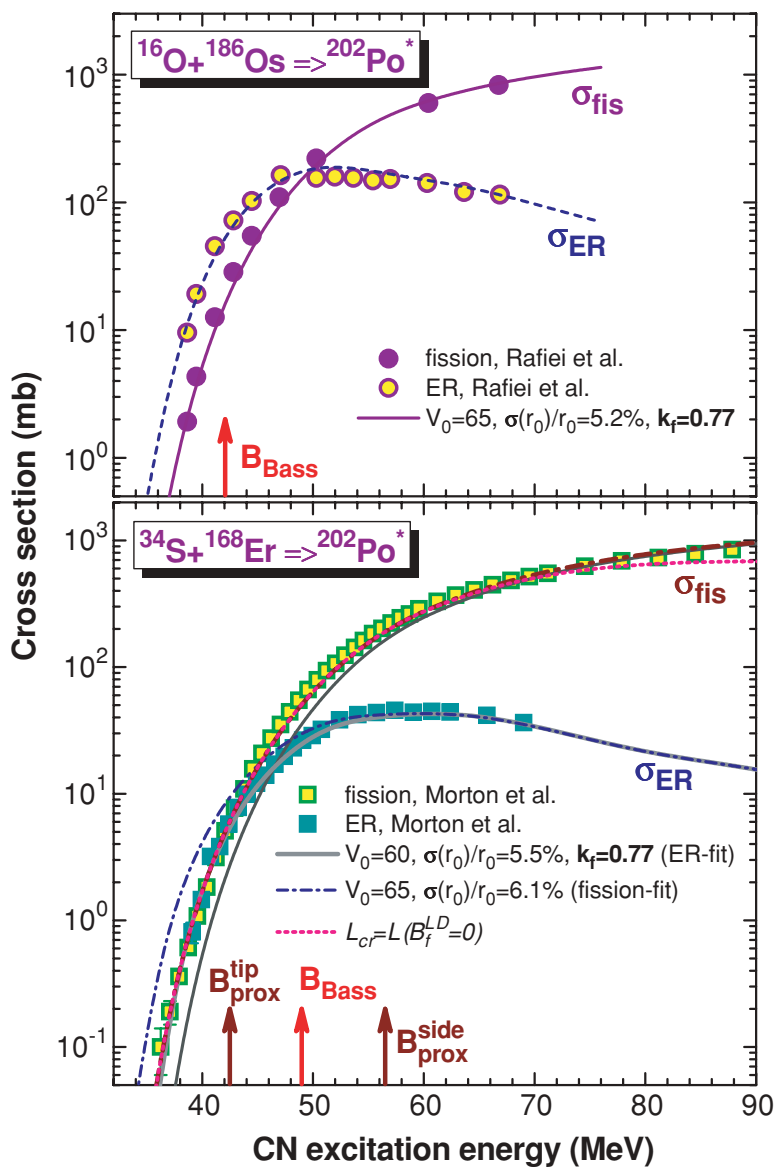

FIG. 2. (Color online) The same as described in the caption to Fig. 1 but for the excitation functions obtained in the ${ }^{16} \mathrm{O}+{ }^{186} \mathrm{Os}$ and ${ }^{34} \mathrm{~S}+{ }^{168} \mathrm{Er}$ reactions [31,32]. Fusion barriers (maxima of the sum of the Coulomb and nuclear proximity potentials) corresponding to different orientations of the deformed target nucleus, i.e., to tip and side configurations [34] are designated by arrows with the $B_{\text {prox }}^{\text {tip }}$ and $B_{\text {prox }}^{\text {side }}$ labels, respectively.

for different projectile-target combinations. The latter varies within $\sigma\left(r_{0}\right) / r_{0}=(5.2-7.5) \%$ as designated in Fig. 1 . Note that the $V_{0}$ value is slightly less than those obtained in the similar analysis of the ${ }^{16,18} \mathrm{O}+\mathrm{Au}$ data [27] $\left(V_{0}=70-75 \mathrm{MeV} / \mathrm{fm}\right)$, whereas the $\sigma\left(r_{0}\right) / r_{0}$ values are higher than those obtained ibidem $\left.\left[\sigma\left(r_{0}\right) / r_{0}=(3.5-4.0) \%\right)\right]$. Such changes correlate with general variations in the potential parameter values at the transition from less deformed (Au) to more deformed (Os) target nuclei.

\section{B. Less asymmetric reactions}

The ${ }^{34} \mathrm{~S}+{ }^{168} \mathrm{Er}$ fission and ER excitation functions obtained by Morton et al. [32] are a convenient starting point in the analysis of less asymmetric reactions, where QF might appear. In Fig. 2 these data are shown together with the ${ }^{16} \mathrm{O}+{ }^{186} \mathrm{Os}$ data [31] leading to the same ${ }^{202} \mathrm{Po}^{*} \mathrm{CN}$ production. The excitation functions measured in the less asymmetric ${ }^{34} \mathrm{~S}+{ }^{168} \mathrm{Er}$ combination are fitted with the same $k_{f}$ value as obtained in the very asymmetric ${ }^{16} \mathrm{O}+{ }^{186} \mathrm{Os}$ one. This is the case at energies well above the nominal fusion 
barrier [22] (irrespectively of the nuclear potential parameters). At sub-barrier and barrier energies the different parameter values of the nuclear potential have to be applied to reproduce the fission and ER excitation functions, as indicated in the bottom panel of the figure. Larger values of $V_{0}$ and $\sigma\left(r_{0}\right) / r_{0}$ for the fission data in comparison with those obtained in a similar fit to the ER ones lead to the lower mean value of the barrier and wider barrier distribution, which can be derived from the corresponding BP cross section. Note that the observation of ER is the unambiguous signature of fusion, whereas fission events might occur both from $\mathrm{QF}$ and a $\mathrm{CN}$ decay. Thus, an excess in the fission cross section (with respect to the calculations corresponding to the ER excitation function fit) observed at sub-barrier and barrier energies can be attributed to the QF effect. This suggestion is supported by the comparison of the measured mass variance and angular anisotropy for fission fragments with the results of transitionstate model calculations [31,32]. The QF effect observed at sub-barrier and barrier energies is usually associated with the orientation effect of a deformed target nucleus [33]. In Fig. 2 the proximity-potential barrier heights corresponding to the tip and side orientations of ${ }^{168} \mathrm{Er}$ in collisions with spherical ${ }^{34} \mathrm{~S}$ (as calculated in Ref. [34]) are shown together the nominal fusion barrier [22]. We emphasize again that this entrance-channel "barrier" effect has no influence on the fission barriers ( $k_{f}$ values) derived in our analysis, if an excitation function well above the nominal fusion barrier is considered, where any "barrier" effects have no meaning.

In Fig. 3 the results of our analysis of the ER cross section data are shown for the ${ }^{27} \mathrm{Al}+{ }^{175} \mathrm{Lu}$ and ${ }^{31} \mathrm{P}+{ }^{169} \mathrm{Tm}$ reactions [35] leading to the ${ }^{202} \mathrm{Po}^{*}$ and ${ }^{200} \mathrm{Po}^{*}$ compound nuclei, respectively. The sum of $x n$ evaporation cross sections $\Sigma \sigma_{x n}$ for the first reaction is well reproduced using the same $k_{f}$ value as in previous cases for ${ }^{16} \mathrm{O}+{ }^{186} \mathrm{Os}$ and ${ }^{34} \mathrm{~S}+{ }^{168} \mathrm{Er}$ leading to the same $\mathrm{CN}$. The production of more neutron-deficient Po nuclei observed in the second reaction corresponds to the slightly lower value of $k_{f}=0.75$. We suppose therewith that fusion suppression does not appear at energies above the Bass barrier in going from the more asymmetric ${ }^{27} \mathrm{Al}+{ }^{175} \mathrm{Lu}$ to less asymmetric ${ }^{31} \mathrm{P}+{ }^{169} \mathrm{Tm}$ reactions. This assumption is supported by the lack of fusion suppression in the more symmetric ${ }^{34} \mathrm{~S}+{ }^{168} \mathrm{Er}$ reaction at energies well above the Bass barrier. Note that the "fusion hindrance" correlates to the position of the entrance-channel mass-asymmetry with respect to the Businaro-Gallone point $[36,37]$ on the plot of the driving potential vs. the mass-asymmetry parameter [18,28].

\section{Symmetric reactions}

The neutron-deficient Po nuclei production in (nearly) symmetric combinations and in reactions with massive projectiles, where the fusion suppression seems to appear, can be considered in terms of the reduced cross sections (see, e.g., $[7,18,25,26])$. It is implied that the sum of the $x n$ evaporation cross sections can be written as

$$
\Sigma \sigma_{x n}=\pi / k^{2} \Sigma(2 L+1) T_{L} P_{\text {fus }}(E, L) W_{x n}\left(E_{\mathrm{CN}}^{*}, L\right),
$$

where $k$ is the wave number, $T_{L}$ is the transmission coefficient for the $L$ partial wave, and $P_{\text {fus }}$ and $W_{x n}$ are the fusion

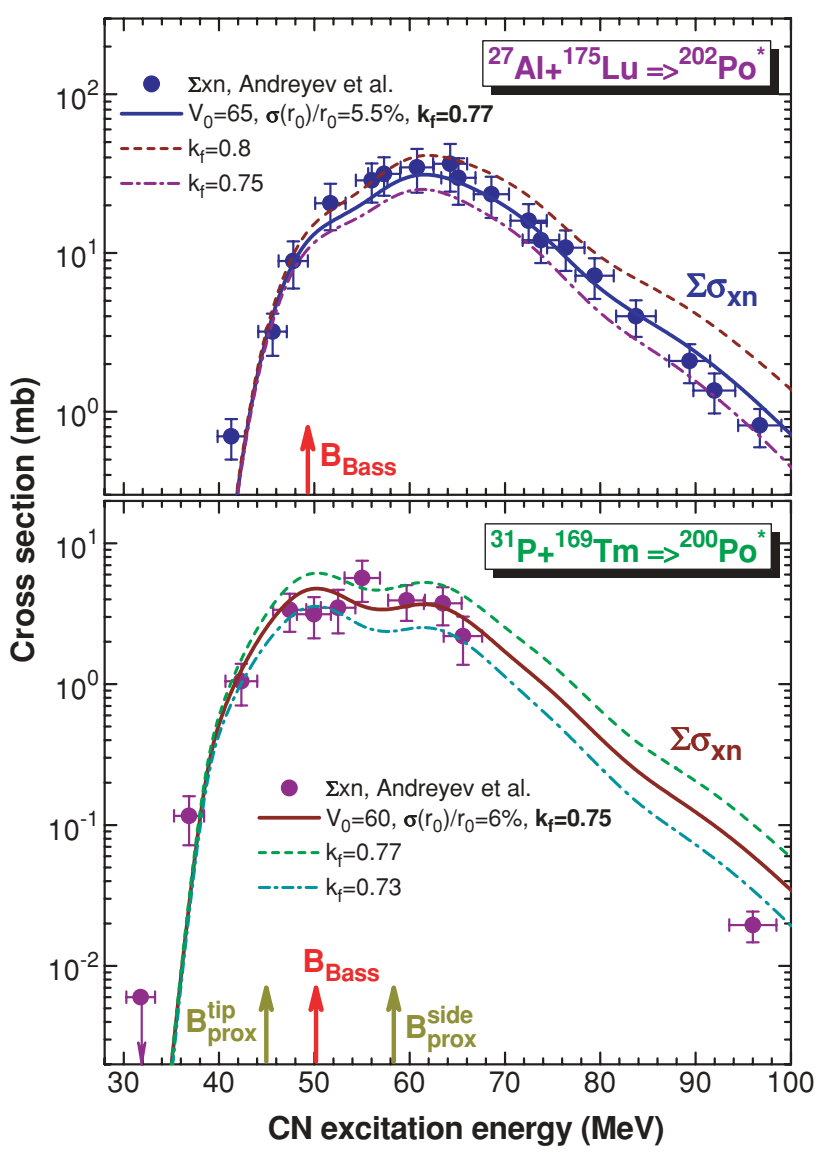

FIG. 3. (Color online) The same as described in the captions Figs. 1 and 2 but for the $\Sigma \sigma_{x n}$ excitation functions obtained in the ${ }^{27} \mathrm{Al}+{ }^{175} \mathrm{Lu}$ and ${ }^{31} \mathrm{P}+{ }^{169} \mathrm{Tm}$ reactions [35]. Calculations correspond to the $L_{\mathrm{cr}}=L\left(B_{f}^{\mathrm{LD}}=0\right)$ restriction.

probability and survivability, respectively. Because the same limited set of angular momenta $(L \lesssim 15 \hbar)$ contributes to the ER production in reactions leading to strongly fissile nuclei, the reduced cross section $\left(k^{2} / \pi\right) \Sigma \sigma_{x n}$ has to be independent of the reaction at a given $\mathrm{CN}$ excitation energy. This is the case at the energy well above the fusion barrier (where $T_{L} \simeq 1$ ), if $P_{\text {fus }}=1$.

In Fig. 4 the reduced cross sections for the Po nuclei produced in the symmetric ${ }^{100} \mathrm{Mo}+{ }^{100} \mathrm{Mo}$ [38] and asymmetric ${ }^{31} \mathrm{P}+{ }^{169} \mathrm{Tm}[35]$ reactions leading to the same ${ }^{200} \mathrm{Po}^{*}$ $\mathrm{CN}$ are compared to the calculations adjusted to the data. The experimental reduced cross sections obtained at $50 \lesssim$ $E_{\mathrm{CN}}^{*} \lesssim 70 \mathrm{MeV}$ for both reactions are nearly the same and are reproduced with the same LD barrier scaling parameter $k_{f}=$ 0.75 . At $E_{\mathrm{CN}}^{*} \lesssim 50 \mathrm{MeV}$ the excitation functions diverge due to the difference in fusion barriers. At $E_{\mathrm{CN}}^{*} \gtrsim 70 \mathrm{MeV}$ the data can be fitted with $k_{f}=0.7$. According to the experiment [38] and our calculations, the evaporation of 6 and 7 neutrons leading to ${ }^{194} \mathrm{Po}$ and ${ }^{193} \mathrm{Po}$, respectively, gives a main contribution to the $\Sigma \sigma_{x n}$ cross section at these energies.

The same $k_{f}$ value is derived when the ${ }^{100} \mathrm{Mo}+{ }^{98} \mathrm{Mo}$ data [38] are fitted at $E_{\mathrm{CN}}^{*} \gtrsim 70 \mathrm{MeV}$ (see Fig. 5). At these energies ${ }^{193} \mathrm{Po}$ produced in the $5 n$ evaporation channel is the main contributor to the $\Sigma \sigma_{x n}$ cross section, according to 


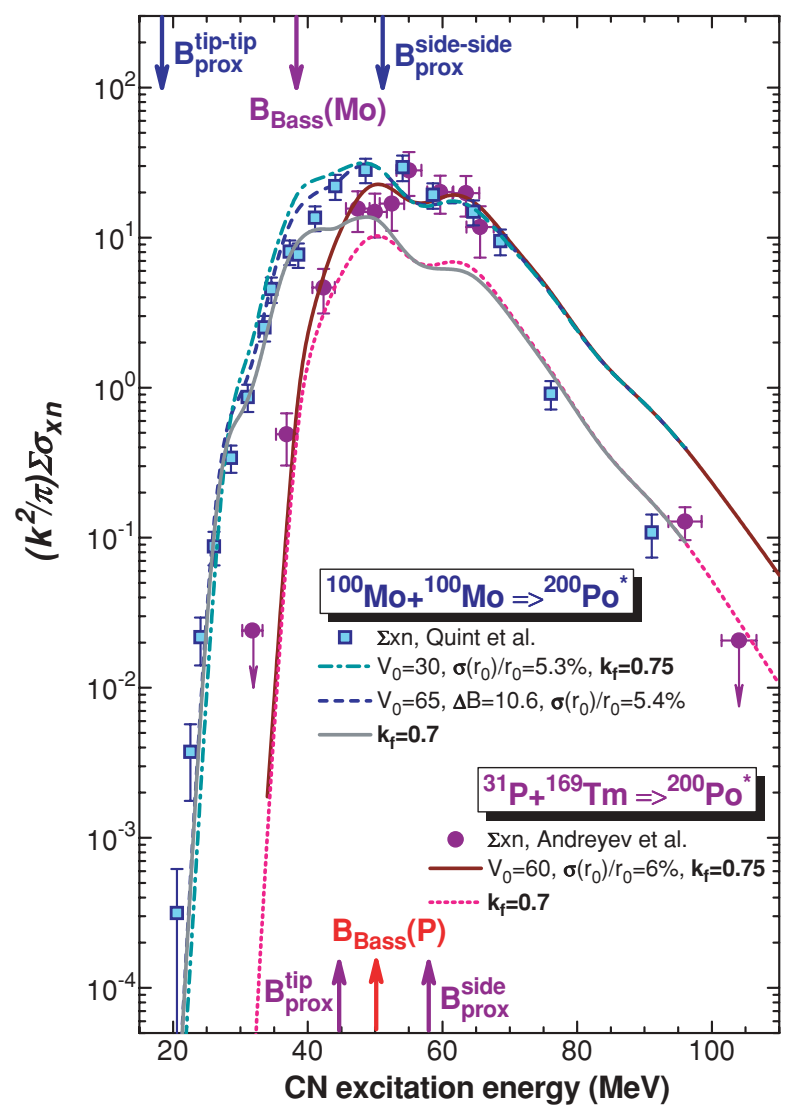

FIG. 4. (Color online) Reduced cross sections for the Po nuclei produced in the symmetric ${ }^{100} \mathrm{Mo}+{ }^{100} \mathrm{Mo}$ [38] and asymmetric ${ }^{31} \mathrm{P}+{ }^{169} \mathrm{Tm}$ [35] reactions leading to the ${ }^{200} \mathrm{Po}^{*} \mathrm{CN}$ (symbols) in comparison with each other and calculations corresponding to the $L_{\mathrm{cr}}=L\left(B_{f}^{\mathrm{LD}}=0\right)$ restriction (lines).

the experiment and our calculations. At $E_{\mathrm{CN}}^{*} \lesssim 70 \mathrm{MeV}$ the excitation function is fitted with $k_{f}=0.72$. The same result is obtained for the ${ }^{58} \mathrm{Fe}+{ }^{140} \mathrm{Ce}$ data [38], as one can see in Fig. 5 .

According to the experiment [38] and our calculations for the ${ }^{100} \mathrm{Mo}+{ }^{96} \mathrm{Mo}$ reaction, ${ }^{193} \mathrm{Po}$ produced in the $3 n$ evaporation channel is the main contributor to the $\Sigma \sigma_{x n}$ cross section at the barrier energies. At these energies the data is quite satisfactory fitted with $k_{f}=0.7$, although the $k_{f}=0.68$ fit is also acceptable because $k_{f}$ strongly correlates with nuclear potential parameters at sub-barrier energies, as one can see in Fig. 6. Note that the height of the fusion barrier corresponding to the "normal" potential with $V_{0}=65 \mathrm{MeV} / \mathrm{fm}$ (this nuclear potential is close to the Bass one [22]) has to be increased by $\Delta B=14 \mathrm{MeV}$ to reproduce the data at sub-barrier and barrier energies. This $\Delta B$ value is greater than similar values obtained in the previous cases for the ${ }^{100} \mathrm{Mo}+{ }^{100} \mathrm{Mo}$ and ${ }^{98} \mathrm{Mo}$ reactions (10.6 and $8.6 \mathrm{MeV}$, respectively, as designated in Figs. 4 and 5). An alternative way to increase the height of the fusion barrier is to reduce $V_{0}$ to $25-35 \mathrm{MeV} / \mathrm{fm}$. Both these approaches have been used to describe the excitation functions obtained in symmetric combinations (see Figs. 4-7 and Sec. IV).

The most neutron-deficient Po isotopes are produced in the ${ }^{52} \mathrm{Cr}+{ }^{142} \mathrm{Nd}[19]$ and ${ }^{100} \mathrm{Mo}+{ }^{94} \mathrm{Mo}$ [38] reactions leading

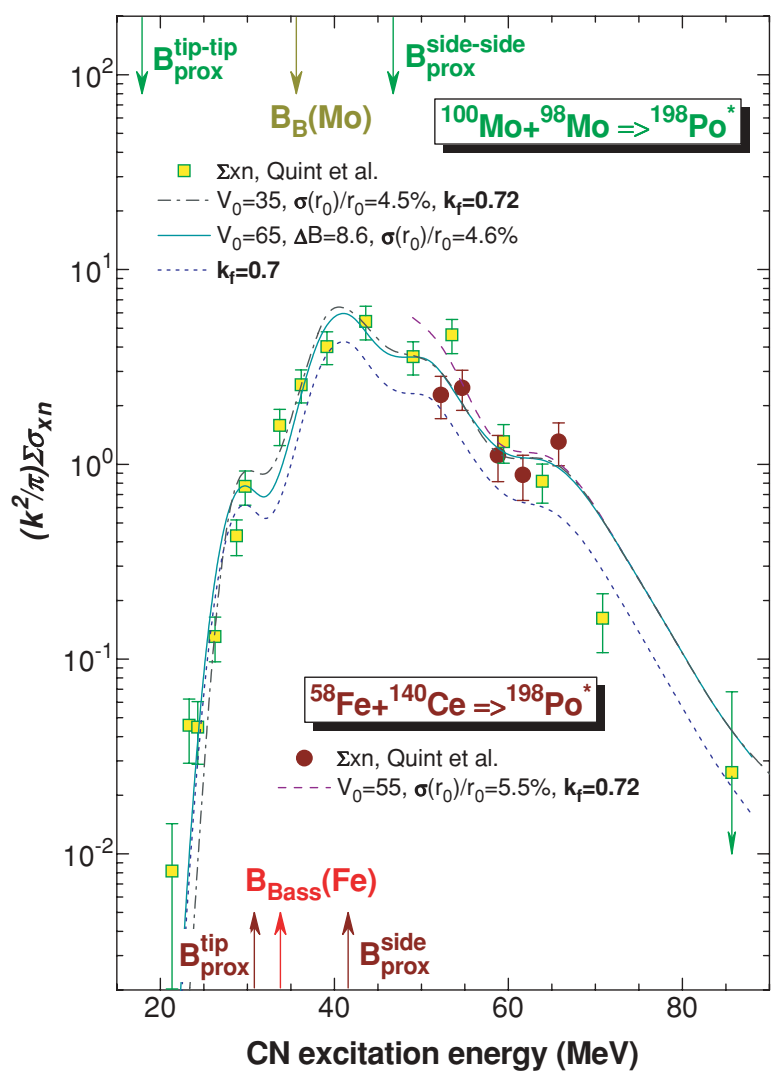

FIG. 5. (Color online) The same as in Fig. 4, but for the ${ }^{100} \mathrm{Mo}+$ ${ }^{98} \mathrm{Mo}$ and ${ }^{58} \mathrm{Fe}+{ }^{140} \mathrm{Ce}$ reactions [38] leading to the ${ }^{198} \mathrm{Po}{ }^{*} \mathrm{CN}$.

to the same ${ }^{194} \mathrm{Po}^{*} \mathrm{CN}$. In the asymmetric combination, the data corresponding to the ${ }^{192-188}$ Po isotopes produced in the (2-6) $n$ evaporation channels can be quite satisfactory fitted with $k_{f}=0.63$, as shown in Fig. 7. In the nearly symmetric ${ }^{100} \mathrm{Mo}+{ }^{94} \mathrm{Mo}$ reaction, the sub-barrier cross section data corresponding to the ${ }^{193-191}$ Po isotopes produced in the (1-3)n evaporation channels can be reproduced with the same $k_{f}$ value, but different nuclear potential parameters. The previous data, corresponding to the ${ }^{198,196} \mathrm{Po}^{*}$ compound nuclei production, show $k_{f}=0.68-0.7$ at the excitation energies well above and close to the fusion barrier, where ${ }^{193} \mathrm{Po}$ and ${ }^{192} \mathrm{Po}$ are the main contributors to the $\Sigma \sigma_{x n}$ cross section (see Figs. 5 and Fig. 6). The production of these nuclei at sub-barrier and barrier energies in the ${ }^{100} \mathrm{Mo}+{ }^{94} \mathrm{Mo}$ and ${ }^{52} \mathrm{Cr}+{ }^{142} \mathrm{Nd}$ reactions can be also reproduced with $k_{f}=0.68$ using the adjustment of nuclear potential parameter values. This adjustment leads to the increase in the height of the fusion barrier (similarly as obtained in the previous cases). Such arbitrariness is due to the correlation between $k_{f}$ and nuclear potential parameters.

\section{DISCUSSION}

\section{A. Fusability in asymmetric and (nearly) symmetric combinations}

The details of the dynamical evolution of a dinuclear system to fusion after the contact of projectile and target nuclei 


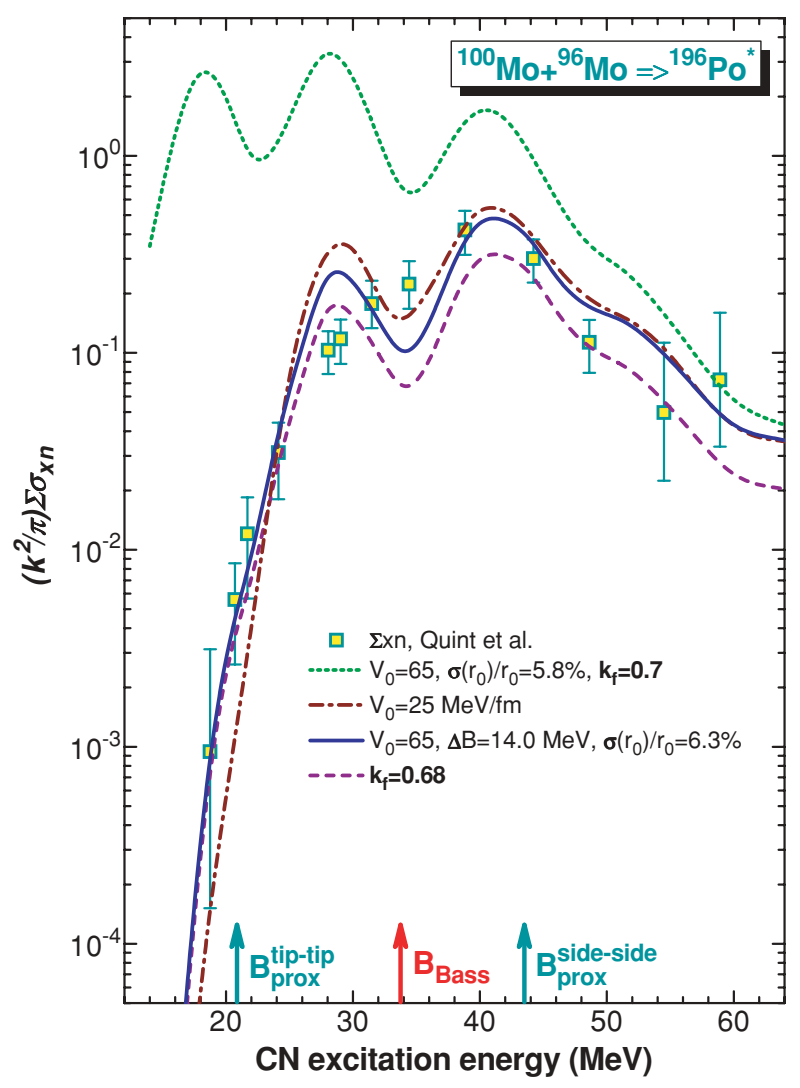

FIG. 6. (Color online) The same as in Figs. 4 and 5, but for the ${ }^{100} \mathrm{Mo}+{ }^{96} \mathrm{Mo}[38]$ reaction leading to the ${ }^{196} \mathrm{Po}^{*} \mathrm{CN}$.

are not well understood at present. Passing the Coulomb barrier and a contact configuration do not guarantee complete equilibration of all degrees of freedom with the formation of a compact mono-nucleus leading to complete fusion. Competing processes like quasifission and deep-inelastic may force the "dinuclear system" to reseparate after large energy losses [11-18], as mentioned in Sec. I. Complete experimental information on these reaction channels is needed to study properly to what extent $\mathrm{CN}$ formation becomes limited. Although the data set is mainly restricted to the ER production (concerning nearly symmetric combinations), in this section we consider consistently the results of the previous section from the point of view of the presence of fusion suppression that can imitate the reduction in the macroscopic component of fission barriers.

Our analysis of the most asymmetric ${ }^{16} \mathrm{O}+{ }^{186} \mathrm{Os}$ reaction shows the same values of the nuclear potential parameters and $k_{f}$ for the description of the ER and fission excitation functions. However, it is not the case for the less asymmetric ${ }^{34} \mathrm{~S}+{ }^{168} \mathrm{Er}$ reaction, as far as the nuclear potential parameters are concerned. As we mentioned in Sec. III, this circumstance is the signature of the QF effect appearing at barrier and subbarrier energies. At energies above the barrier the ${ }^{34} \mathrm{~S}+{ }^{168} \mathrm{Er}$ ER and fission excitation functions are fitted with the same $k_{f}$ value (irrespectively of the potential parameters) as obtained in the ${ }^{16} \mathrm{O}+{ }^{186} \mathrm{Os}$ data analysis (see Fig. 2). Being exclusively an entrance-channel effect, QF in the ${ }^{34} \mathrm{~S}+{ }^{168} \mathrm{Er}$ reaction does not distort the fission barriers derived from the data. These data

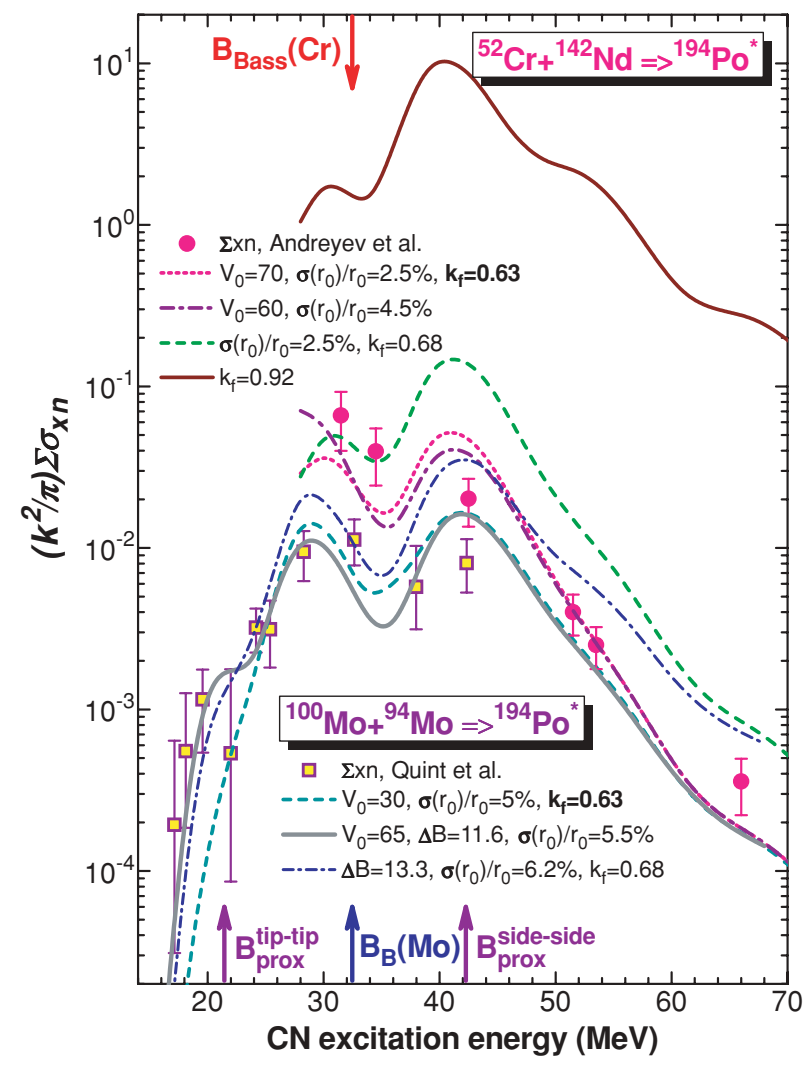

FIG. 7. (Color online) The same as in Figs. 4-6, but for the ${ }^{100} \mathrm{Mo}+{ }^{94} \mathrm{Mo}$ [38] and ${ }^{52} \mathrm{Cr}+{ }^{142} \mathrm{Nd}$ [19] reactions leading to the ${ }^{194} \mathrm{Po}^{*} \mathrm{CN}$.

allow us to evaluate a fusion probability function as the ratio of the measured ER cross sections and the calculated ones corresponding to the fission excitation function fit (see Fig. 8).

The analysis of the ${ }^{27} \mathrm{Al}+{ }^{175} \mathrm{Lu}$ excitation function shows the same $k_{f}$ value as obtained in the previous cases corresponding to the production of the same ${ }^{202} \mathrm{Po}^{*} \mathrm{CN}$ in more and less asymmetric combinations. The data allow one to estimate fission barriers for the nuclei of an evaporation chain up to ${ }^{194} \mathrm{Po}$ produced in the $8 n$ evaporation channel at the highest excitation energies. The same nuclide is produced in the $6 n$ evaporation channel of the less asymmetric ${ }^{31} \mathrm{P}+$ ${ }^{169} \mathrm{Tm}$ reaction leading to the ${ }^{200} \mathrm{Po}^{*} \mathrm{CN}$. Similar values of $k_{f}=0.75-0.77$ used to describe the ${ }^{194}$ Po production in ${ }^{175} \mathrm{Lu}\left({ }^{27} \mathrm{Al}, 8 n\right)$ and ${ }^{169} \mathrm{Tm}\left({ }^{31} \mathrm{P}, 6 n\right)$ within the cross-section error bars (see Fig. 3) allow us to state that QF has a minor effect on the Po nuclei production in the ${ }^{31} \mathrm{P}+{ }^{169} \mathrm{Tm}$ reaction, at least at energies above the nominal fusion barrier.

The last statement enables to use ${ }^{31} \mathrm{P}+{ }^{169} \mathrm{Tm}$ as a reference reaction for the evaluation of the fusion probability function in the symmetric ${ }^{100} \mathrm{Mo}+{ }^{100} \mathrm{Mo}$ combination. Nearly the same reduced ER cross sections for both reactions at energies $50 \lesssim E_{\mathrm{CN}}^{*} \lesssim 70 \mathrm{MeV}$, which are well reproduced with the same $k_{f}=0.75$ (see Fig. 4), imply that $P_{\text {fus }} \simeq 1$ in this energy range. At higher energies the drop in the ER cross sections is caused by the noticeable decrease in the LD fission barriers, as considered in Sec. III. At lower energies the Po nuclei production in ${ }^{100} \mathrm{Mo}+{ }^{100} \mathrm{Mo}$ is suppressed by the fusion 


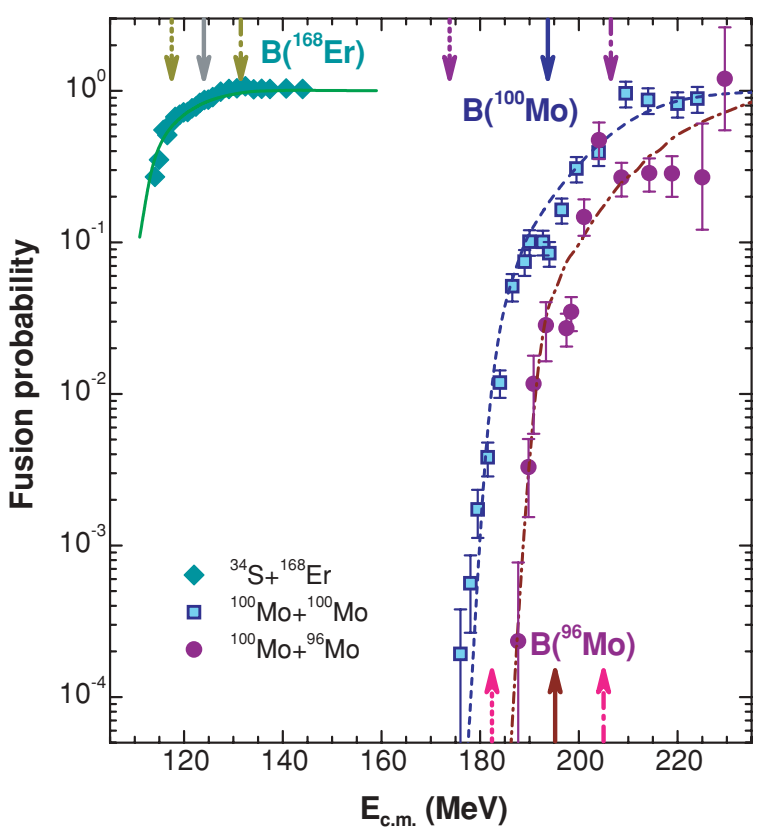

FIG. 8. (Color online) Fusion probability functions derived empirically for the asymmetric ${ }^{34} \mathrm{~S}+{ }^{168} \mathrm{Er}$ and (nearly) symmetric ${ }^{100} \mathrm{Mo}+{ }^{96,100}$ Mo systems (symbols) in comparison with the ones obtained in calculations (lines). Nominal fusion barriers [22] are shown with solid arrows, whereas the barriers obtained with the proximity potential, taking into account the deformation for tip-tip and side-side collisions [34], are shown with dotted and dash-dotted arrows, respectively.

barrier, which is significantly higher than the expected one, if $V_{0}=65 \mathrm{MeV} / \mathrm{fm}$ is used. To reproduce the Po production cross sections at these energies the expected fusion barrier has to be increased by $\Delta B=10.6 \mathrm{MeV}$. Alternatively, much smaller value of the nuclear strength parameter $\left(V_{0}=30 \mathrm{MeV} / \mathrm{fm}\right)$ has to be used. The example of the "unsuppressed" Po cross section calculation with $V_{0}=65 \mathrm{MeV} / \mathrm{fm}$ is shown for the ${ }^{100} \mathrm{Mo}+{ }^{96} \mathrm{Mo}$ reaction in Fig. 6. The similar calculation for ${ }^{100} \mathrm{Mo}+{ }^{100} \mathrm{Mo}$ allows us to evaluate the fusion probability function in the whole range of energies (see Fig. 8).

At $E_{\mathrm{CN}}^{*} \gtrsim 70 \mathrm{MeV}$ both the ${ }^{200} \mathrm{Po}^{*}$ and ${ }^{198} \mathrm{Po}^{*} \mathrm{CN}$ data can be fitted with $k_{f}=0.7$ (see Figs. 4 and 5). At these energies ${ }^{193} \mathrm{Po}$ is mainly produced in the $7 n$ and $5 n$ evaporation channels on the ${ }^{100} \mathrm{Mo}$ and ${ }^{98} \mathrm{Mo}$ target nuclei, respectively. The production of this nucleus in the $3 n$ evaporation channel on the ${ }^{96}$ Mo target nucleus at $E_{\mathrm{CN}}^{*} \lesssim 50 \mathrm{MeV}$ can be satisfactory reproduced with the similar barrier scaling $\left(k_{f}=0.68\right)$ and nuclear potential parameter values, as obtained in the cases of symmetric reactions leading to ${ }^{200,198} \mathrm{Po}^{*}$ (see Fig. 6). As one can see in Fig. 8, the fusion probability function obtained for the ${ }^{100} \mathrm{Mo}+{ }^{96} \mathrm{Mo}$ reaction, in the same way as in the previous case for ${ }^{100} \mathrm{Mo}+{ }^{100} \mathrm{Mo}$, is very similar to the one derived for the latter, but without any normalization on asymmetric cross-section data.

Reduced cross sections obtained experimentally in reactions leading to the ${ }^{194} \mathrm{Po}^{*} \mathrm{CN}$ do not converge up to $E_{\mathrm{CN}}^{*} \simeq$ $42 \mathrm{MeV}$, the highest common point on the energy for both the asymmetric ${ }^{52} \mathrm{Cr}+{ }^{142} \mathrm{Nd}$ and nearly symmetric ${ }^{100} \mathrm{Mo}+$
${ }^{94}$ Mo reaction data (see Fig. 7). With the corresponding choice of the nuclear potential parameters one can reproduce the cross sections obtained at barrier and sub-barrier energies in the asymmetric and nearly symmetric combinations, respectively. These data correspond to the ${ }^{192,193}$ Po production and can be reproduced with $k_{f}=0.68$, i.e., with the LD barrier scaling that characterizes the production of these nuclei in reactions leading to the ${ }^{198,200} \mathrm{Po}^{*}$ compound nuclei at higher energies. At energies above the fusion barrier $\left(E_{\mathrm{CN}}^{*}>40 \mathrm{MeV}\right)$, the cross sections for the ${ }^{188-191} \mathrm{Po}$ production in ${ }^{52} \mathrm{Cr}+{ }^{142} \mathrm{Nd}$ are better described with $k_{f}=0.63$, as one can see in Figs. 7 and 10. Calculations show the convergence of the reduced cross sections for both reactions at $E_{\mathrm{CN}}^{*} \gtrsim 65 \mathrm{MeV}$ ( $P_{\text {fus }}=1$ is assumed), as shown in Fig. 7.

Summarizing the consideration of fusability in asymmetric and (nearly) symmetric combinations leading to the production of Po nuclei, one can conclude that the fusion suppression observed at barrier and sub-barrier energies can be taken into account in the analysis of the ER excitation functions obtained in experiments. The entrance-channel effect of the fusion suppression cannot significantly garble information on the LD fission barriers derived from the present analysis.

\section{B. Macroscopic fission barriers for Po nuclei}

The reduced LD (macroscopic) fission barriers for Po nuclei resulting from the present analysis are shown in Fig. 9. In the cases of the ${ }^{204,206,208,210} \mathrm{Po}^{*} \mathrm{CN}$ data obtained in the ${ }^{16,18} \mathrm{O}+{ }^{188,190,192} \mathrm{Os}$ reactions, we limited the consideration by the region of $E_{\mathrm{CN}}^{*} \lesssim 100 \mathrm{MeV}$. At higher energies the fission cross section becomes less sensitive to the variations of fission barriers in going to more neutron-deficient (thus, more fissile) compound nuclei (see Fig. 1). This limit on the energy is close to the position of maxima of the $9 n$ and $8 n$ evaporation channels for the ${ }^{206,208,210} \mathrm{Po}^{*}$ and ${ }^{204} \mathrm{Po}^{*}$ compound nuclei, respectively. The cross-section data for the ${ }^{202} \mathrm{Po}^{*} \mathrm{CN}$ are obtained in three reactions $\left({ }^{16} \mathrm{O}+{ }^{186} \mathrm{Os}\right.$, ${ }^{27} \mathrm{Al}+{ }^{175} \mathrm{Lu}$, and ${ }^{34} \mathrm{~S}+{ }^{168} \mathrm{Er}$ ) up to the energy of $E_{\mathrm{CN}}^{*} \simeq$ $100 \mathrm{MeV}$ (see Figs. 2 and 3). Near this energy the $8 n$ evaporation cross section obtained in Refs. [35] is well reproduced in our calculations with $k_{f}=0.75-0.77$. The analysis shows the same $k_{f}$ value for both the ${ }^{31} \mathrm{P}+{ }^{169} \mathrm{Tm}$ and ${ }^{100} \mathrm{Mo}+{ }^{100} \mathrm{Mo}$ reactions (leading to the ${ }^{200} \mathrm{Po}^{*} \mathrm{CN}$ ) at $E_{\mathrm{CN}}^{*} \lesssim 70 \mathrm{MeV}$. At these energies the $6 n$ evaporation cross section measured in Refs. [35,38] is well reproduced with $k_{f}=0.75$ in our calculations. The similar approach is applied to the ${ }^{198} \mathrm{Po}^{*} \mathrm{CN}$ data obtained in two reactions $\left({ }^{58} \mathrm{Fe}+{ }^{140} \mathrm{Ce}\right.$ and $\left.{ }^{100} \mathrm{Mo}+{ }^{98} \mathrm{Mo}\right)$, which are well reproduced in our calculations with $k_{f}=0.72$ up to the energy of $E_{\mathrm{CN}}^{*} \simeq 65 \mathrm{MeV}$, i.e., up to the maximum of the $5 n$ evaporation channel. The ${ }^{100} \mathrm{Mo}+{ }^{96} \mathrm{Mo}$ cross-section data are limited by the energy of $E_{\mathrm{CN}}^{*} \simeq 60 \mathrm{MeV}$ and by the observation of the $4 n$ evaporation channel. The data can be reproduced with $k_{f}=0.68-0.7$ in the calculations. The ${ }^{100} \mathrm{Mo}+{ }^{98} \mathrm{Mo}$ data at $E_{\mathrm{CN}}^{*}>65 \mathrm{MeV}$ indicate the same $k_{f}$ value (see Figs. 5 and 6). The ${ }^{194} \mathrm{Po}^{*} \mathrm{CN}$ data obtained at $E_{\mathrm{CN}}^{*}<36 \mathrm{MeV}$ correspond to the ${ }^{193,192}$ Po production and allow one a description with $k_{f}=0.68$, i.e., with the same value that is used for the description of the ${ }^{196} \mathrm{Po}^{*}$ and ${ }^{198} \mathrm{Po}^{*}$ 


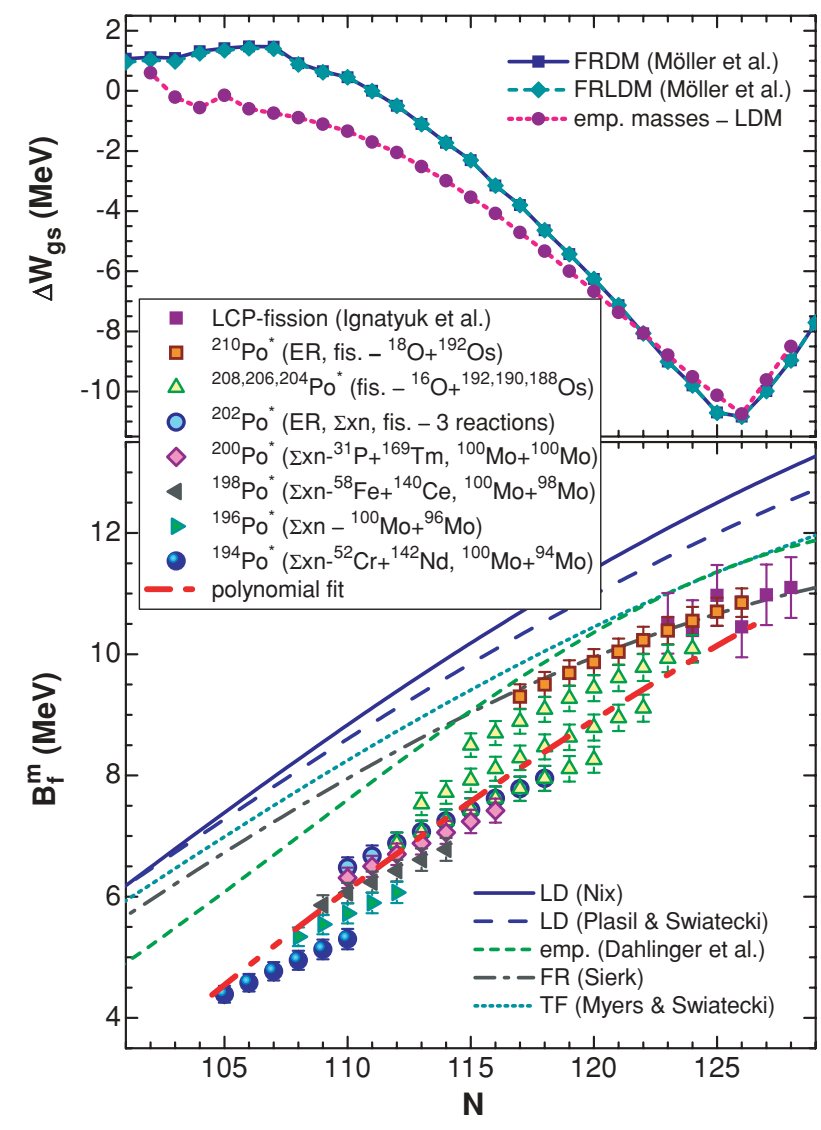

FIG. 9. (Color online) The bottom panel shows macroscopic fission barriers resulting from the analysis of fusion reactions leading to Po nuclei (open symbols) in comparison to various theoretical model predictions [2-4,40,41] (lines) and to the results obtained in light-charged-particles induced fission [39] (solid squares). Thick dash-double-dotted line corresponds to the polynomial fit to the data derived in the present work. The upper panel shows ground-state shell corrections predicted in Ref. [5] and those used in the present work.

$\mathrm{CN}$ data at $E_{\mathrm{CN}}^{*} \lesssim 60 \mathrm{MeV}$ and $E_{\mathrm{CN}}^{*}>65 \mathrm{MeV}$, respectively. The ${ }^{194} \mathrm{Po}^{*} \mathrm{CN}$ data obtained at $E_{\mathrm{CN}}^{*}>36 \mathrm{MeV}$ correspond to the ${ }^{188-191}$ Po production and can be only reproduced with the smaller value of $k_{f}=0.63$ (see Figs. 7 and 10). The uncertainties in the scaling parameter values are estimated to be $\delta k_{f} \simeq \pm 0.02$ so $\left(\delta k_{f} / k_{f}\right)$ values approximately reproduce the relative cross-section errors and follow the tendency to their increase with the neutron deficiency of nuclei produced in the reactions.

In Fig. 9 macroscopic fission barriers derived from the present study are compared with those obtained earlier in light-charged-particles induced fission [39] and with the macroscopic barriers predicted by various theoretical and empirical models [2-4,40,41]. In the upper panel of the figure, ground-state shell corrections used in the present analysis (see Sec. II) are shown as well as the ones obtained in the framework of the finite-range model (droplet and liquid-drop) [5]. The former values were applied to the data [39]. According to any theory, macroscopic fission barriers should be a smooth function of $N$. We remind that in our SSM analysis the $k_{f}$-scaling is the same for all nuclei in the de-excitation cascade of a given $\mathrm{CN}$. Therefore, if the $k_{f}$ values are different, we have to obtain a stepwise change in the heights of the barriers for different $\mathrm{CN}$ masses, as we see in Fig. 9. A polynomial fit to the data demonstrates significantly smaller macroscopic fission-barrier heights than any predictions for neutron-deficient Po nuclei. At the same time the macroscopic fission barriers derived in the region around $N=126$ are in good agreement with the ones obtained in Ref. [39] and with the values calculated in the framework of the finite-range model [3]. Note that in the region of spherical nuclei, the shell correction energies used in the present analysis $\Delta W_{\mathrm{gs}}^{\mathrm{emp}}$ (see Sec. II) are similar to the microscopic energy $\Delta W_{\mathrm{gs}}^{\mathrm{FRD}}$ calculated with the finite-range model [5], whereas in the region of neutron-deficient nuclei with $105 \leqslant N \leqslant 110$ the difference between mean values is $S_{0}=\left\langle\Delta W_{\mathrm{gs}}^{\mathrm{FRD}}\right\rangle-\left\langle\Delta W_{\mathrm{gs}}^{\mathrm{emp}}\right\rangle=$ $1.87 \mathrm{MeV}$ (see the upper panel in Fig. 9). Because the shell corrections are the input parameters of our SSM analysis, the final values of the macroscopic component of fission barrier are sensitive to their choice.

We studied the effect of different shell correction values (microscopic component) on the final values of the macroscopic barriers in more detail. The comparison of the empirical masses [23] with the calculated ones [5] in the region of neutron-deficient nuclei with $105 \leqslant N \leqslant 115$ shows that the mean deviation of the finite-range droplet masses from the empirical ones is $-0.05 \mathrm{MeV}$, whereas the similar value for the finite-range $\mathrm{LD}$ masses is $0.873 \mathrm{MeV}$. Therefore, using the finite-range droplet masses and shell corrections determined by the difference between these masses and LD masses, we have to obtain the final macroscopic fission barriers very close to the ones shown in Fig. 9. Their magnitudes are independent of the macroscopic (LD [1] or finite-range [3]) barriers, which are used as input values in the analysis. To estimate the effect of the shell correction on the macroscopic component resulting from the analysis, the ground-state shell correction values used in the ${ }^{52} \mathrm{Cr}+{ }^{142} \mathrm{Nd}$ cross-section calculations were increased by the value of $S_{0}=1.87 \mathrm{MeV}$ (the same one for all nuclei involved in the de-excitation cascade). This allows one to imitate the calculation with the finite-range droplet masses and corresponding shell corrections [5]. The results are shown in Fig. 10 for the modified shell corrections (these values became appreciably positive) and the $k_{f}$ value adjusted to reproduce the $x n$ cross-section data [19]. As we see, the calculated excitation functions are comparable with the previous ones using the empirical masses [23] and the ground-state shell corrections as the difference between these and LD masses [24] (see Sec. II). In other words, both calculations, either with the "small" (negative) or "large" (positive) shell corrections, reproduce reasonably well the evaporation cross sections using quite different scaling parameter values of either $k_{f}=0.63$ or 0.73 , respectively. Note that the total heights of fission barriers in both calculations are about the same. It means that the accuracy in the derivation of the macroscopic fission barriers is limited by our knowledge of the ground-state shell correction energy.

\section{Search for the CELD effect}

As mentioned in Sec. I, according to the studies [9,10], the strongest manifestation of the collective enhancement in 


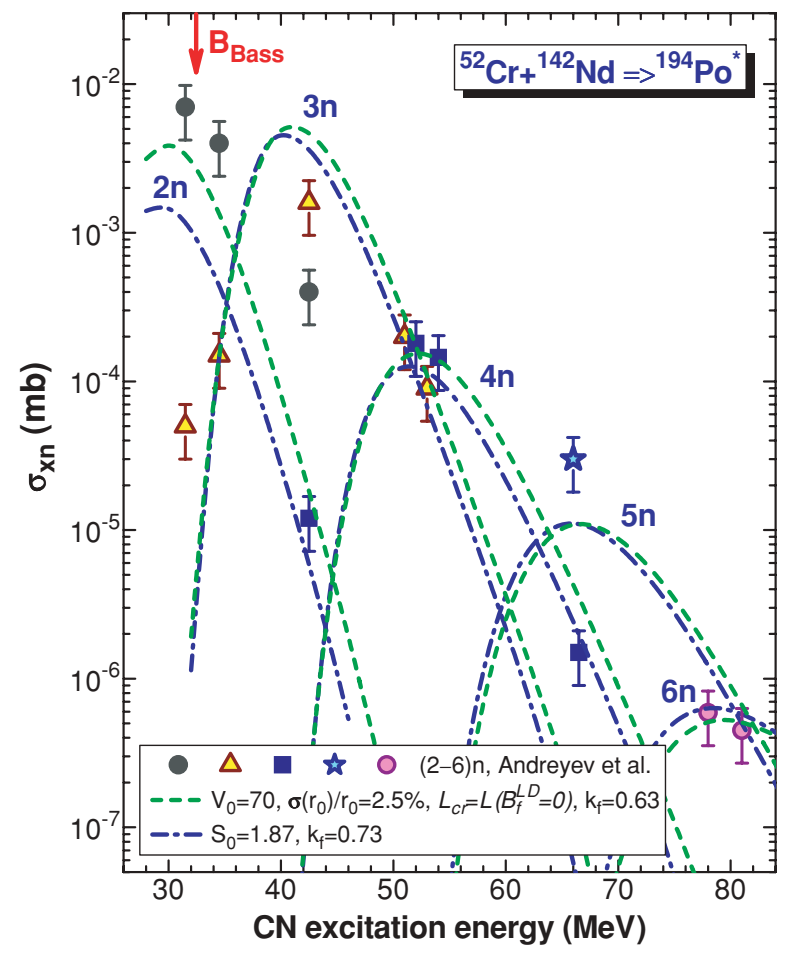

FIG. 10. (Color online) Evaporation cross sections for the Po nuclei produced in the ${ }^{142} \mathrm{Nd}\left({ }^{52} \mathrm{Cr}, x n\right)$ reactions [19] (symbols) in comparison to the calculations using different components of macroscopic fission barriers and ground-state shell corrections (lines) as described in the text.

the nuclear level density are expected in the production of spherical Po nuclei in the vicinity of $N=126$. In our SSM analysis of the ${ }^{18} \mathrm{O}+{ }^{192} \mathrm{Os}$ cross-section data $[29,30]$, we could reproduce them in calculations using the macroscopic fission barriers, which are essentially the same as those derived in the analysis of fissility of Po nuclei in reactions induced by light charged particles [39]. Nearly the same values are predicted by the finite-range model calculations [3] (see Figs. 1 and 9). Note that the shell corrections used in our analysis and calculated in the framework of the finite-range model [5] are about the same in the region of $117 \leqslant N \leqslant 126$ (see Fig. 9), so these values cannot significantly change the heights of macroscopic fission barriers derived with our approach. In going to the neutron-deficient nuclei, the macroscopic component of fission barriers becomes significantly lower than any predictions (see Fig. 9). In fact, this lowering reflects a severe decrease in the production cross sections of Po nuclei, as it follows from the results of the present analysis. One may inversely say that the low production cross sections are a consequence of the lower barriers. In Fig. 7, we show the reduced cross section calculated with $k_{f}=0.92$ that corresponds to the finite-range barriers [3] (the same value is used for the description of the spherical nuclei production in the ${ }^{18} \mathrm{O}+{ }^{192} \mathrm{Os}$ reaction). As one can see, this calculation exceeds the ${ }^{52} \mathrm{Cr}+{ }^{142} \mathrm{Nd}$ cross sections by about three orders of magnitude. At the same time, according to the schematic model [10] one could expect even higher production cross

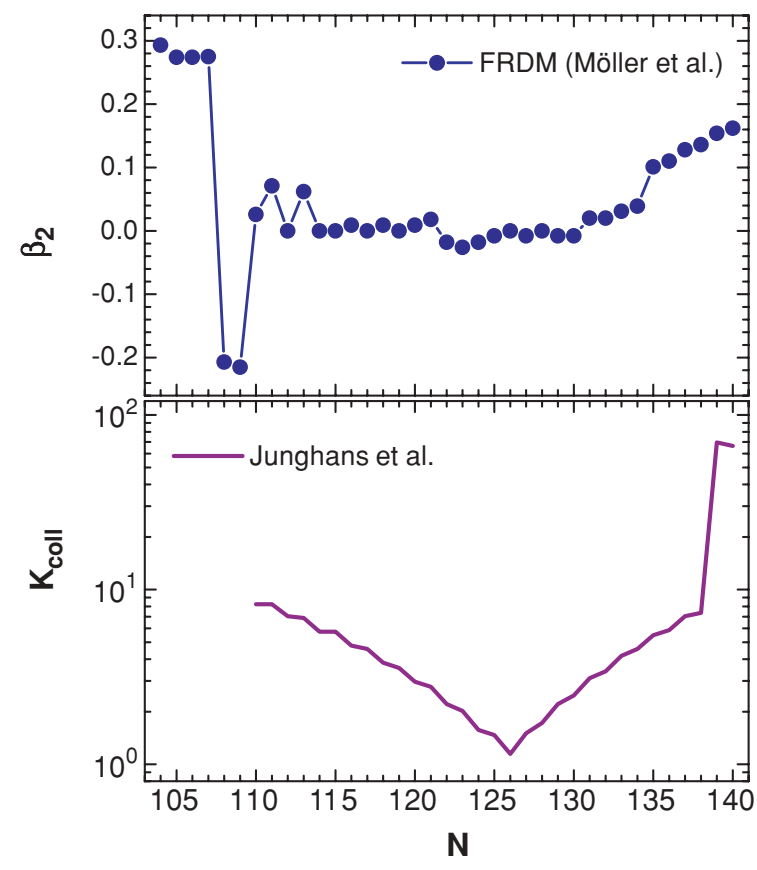

FIG. 11. (Color online) $\beta_{2}$ quadrupole deformations for Po nuclei as calculated in the framework of the finite-range droplet model [5] (upper panel) and the collective enhancement in their level density according to [10] (bottom panel).

sections for the well-deformed Po nuclei with $N<110$ than those obtained in calculations with $k_{f}=0.92$.

Let us consider the last statement in more detail. According to Refs. $[9,10]$ the total level density $\rho\left(E^{*}\right)$ can be expressed as a product of $\rho_{\text {intr }}\left(E^{*}\right) K_{\text {coll }}\left(E^{*}\right)$, i.e., the level density of intrinsic excitations $\rho_{\text {intr }}\left(E^{*}\right)$ and a collective enhancement factor $K_{\text {coll }}\left(E^{*}\right)$. The collective enhancement factor is shown in Fig. 11 (bottom panel), as obtained in the schematic model [10] for Po nuclei. According to this model, there is a sharp transition in $K_{\text {coll }}\left(E^{*}\right)$ from the rotational enhancement of $K_{\text {rot }} \simeq 70$ for deformed Po nuclei with $N>138$ to the vibrational enhancement of $K_{\mathrm{vib}} \simeq 1-10$ for near spherical nuclei with $N=110-138$. The enhancement factor $K_{\text {rot }}$ is independent of the deformation value and is about the same in the fission (saddle point) and evaporation (equilibrium point) channels and therefore it has no influence on the survivability of deformed Po nuclei. At the same time, the fact that $K_{\text {rot }}$ in the fission channel is not compensated by $K_{\text {vib }}$ in the evaporation channel for spherical nuclei dramatically decreases their survivability [10]. We remind that only intrinsic excitations are considered in the SSM [6] used in our analysis. Therefore, describing the spherical nuclei production in our model, we should obtain the cross-section values, which are much higher than those calculated with the use of nominal fission barriers (according to the finite-range model [3] or to the LD one [1,41] with the $k_{f}=0.92$ scaling), if the CELD effect really occurs in the $\mathrm{CN}$ de-excitation. However, it is not the case, because one can describe the production of spherical Po nuclei in the region of $110 \leqslant N \leqslant 126$ in the framework of SSM [6], starting with the nominal heights of macroscopic fission barriers and followed by decrease in their heights with 
the decrease of $N$, without taking into account the collective enhancement in the level density. Moreover, considering the well-deformed Po nuclei in the region of $104 \leqslant N<110$, similar calculations show the further consistent decrease in the macroscopic fission barriers, which corresponds to the further drop in the production cross section observed in the experiment [19]. At the same time, one can expect from the schematic model [10] that the transition from spherical to deformed Po nuclei must be accompanied by a relative increase in the production cross section (due to the corresponding increase in the $K_{\text {rot }}$ enhancement factor in the evaporation channel), which is not observed in the experiment [19].

Such nonobservation of the collective enhancement in the level density is not quite unexpected. It is well known (see, e.g., Ref. [39] and references therein) that the most reliable information on absolute nuclear level densities originates from counting low-lying levels and from neutron resonances. A quantitative analysis of the neutron-resonance data revealed the contribution of collective excitations at excitation energies around $7 \mathrm{MeV}$ [42]. The analysis of the fragmentation cross sections also reveals their strong sensitivity to the collective properties of the nuclear level density [10]. At the same time, the data analysis of light charged particles induced fission in the $\mathrm{Pb}$ region shows that the quite good description of fission excitation functions can be similarly achieved both with the inclusion of the collective excitations [39] and without them [43]. In the later case a three-parameter fit is used with the macroscopic level-density parameter ratio $\tilde{a}_{f} / \tilde{a}_{n}$, an effective fission barrier $B_{f}^{*}$, and the ground-state shell effect for the daughter nucleus after neutron evaporation as fitting variables. The values of $\tilde{a}_{f} / \tilde{a}_{n}$ derived in the analysis of excitation functions for Po compound nuclei [43] are about the same as those obtained in Ref. [39] for similar experimental data. The ground-state shell effect is close to the one calculated with the finite-range droplet model [5], whereas the $B_{f}^{*}$ values for Po nuclei are noticeably higher than the finite-range barriers [3] (corrected with the ground-state shell effect [5]) and those obtained in Ref. [39] (even with taking into account corrections on the pairing condensation energy [43]).

Mention should be made of the recent analysis of the crosssection data obtained in the $\mathrm{O}+\mathrm{Pb}$ and $\mathrm{Ar}+\mathrm{Hf}$ reactions leading to the Th ER produced around the $N=126$ shell [44]. It shows that a decrease in the production cross section for spherical Th nuclei, which can be assigned to the CELD effect, does not exceed the factor of 3-5. This is much smaller than one could expect according to the prediction of the schematic model [10]. The comparison of the ER cross sections for the very asymmetric ${ }^{16} \mathrm{O}+{ }^{204} \mathrm{~Pb}$ and less asymmetric ${ }^{40} \mathrm{Ar}+{ }^{180} \mathrm{Hf}$ reactions leading to the same $\mathrm{CN}$ shows severe inhibition for fusion for the latter $\left(P_{\text {fus }}=0.25\right)$. It implies that the drop in production cross sections of Th nuclei in the vicinity of $N=126$ (see Sec. I) is mainly caused by the entrance-channel QF effect [44]. It should be also mentioned that no signature of the CELD was revealed in the analysis of the low-energy heavy-ion experiment on the detection of evaporative $\alpha$ particles from the ${ }^{178} \mathrm{Hf}^{*} \mathrm{CN}$ [45]. The data are best reproduced by calculations that do not explicitly consider the collective enhancement and its fading out.

\section{SUMMARY}

The ER and fission excitation functions measured in various complete fusion reactions leading to the Po compound nuclei in the mass region from $A_{\mathrm{CN}}=210$ to 194 have been analyzed in the framework of the standard statistical model approximations [6]. The data can be reproduced in calculations using the LD fission barriers scaling applied earlier in similar considerations of the neutron-deficient Po nuclei production [19]. The $A_{\mathrm{CN}}=$ 210 fission and ER excitation functions are well described with the macroscopic component of fission barriers similar to the one calculated in the framework of the finite-range model [3]. The analysis of the $A_{\mathrm{CN}}<210$ data demonstrates a gradual decrease in the macroscopic component of fission barrier with a neutron number, which is stronger than the predictions of any theoretical or empirical model $[2-4,40,41]$. The $A_{\mathrm{CN}}=$ 194 cross-section data [19] show significant reduction in the macroscopic component of fission barriers, amounting to $63 \%$ of the nominal LD values $[1,41]$. This result is close to the one obtained in the preceding study [19].

Reduction in the absolute values of the macroscopic fission barriers derived with the present analysis are determined by fixing the remaining parameters of SSM, such as the $\tilde{a}_{f} / \tilde{a}_{v}$ values, the damping constant in fading out of the shell effect with an excitation energy, and the values of the moment of inertia at the equilibrium state and saddle point [6]. We showed that uncertainties in the absolute magnitudes of the macroscopic fission barriers, which mainly reflect the errors in the ER cross section values used for the estimates, comprise a minor part of the total error. Main uncertainties come from the lack of our knowledge of the ground-state shell correction energies for well-deformed nuclei.

The analysis of the fusability in (nearly) symmetric reactions leading to the neutron-deficient Po nuclei shows that the fusion suppression, which might imitate the reduction observed in the LD fission barriers [19], only appears at sub-barrier and barrier energies. It can be taken into account with the corresponding adjustment of the nuclear potential parameters leading to the significant increase in the fusion barrier height. At the same time, the LD fission barrier scaling derived from the ER cross-section data obtained at energies well above the fusion barrier is in line with the one derived from the data analysis of more asymmetric reactions or/and reactions leading to more neutron-rich compound nuclei.

The search for the manifestation of collective enhancement in the level density of de-exciting Po nuclei, which might strongly modulate the ER production in going from spherical to deformed nuclei (according to the considerations proposed in Ref. $[9,10])$, has not been successful in the present study using the SSM approximations [6]. The gradual drop in the production cross sections of Po nuclei in going from spherical with $N=126$ to well-deformed ones with $N<110$, which is reflected in the decrease in the macroscopic component of fission barriers, does not correspond to the expectations related to the collective excitations and deformation of nuclei involved in a $\mathrm{CN}$ de-excitation $[9,10]$.

A similar study of heavier nuclei production in a wide region of $N$ is of interest from the point of view of the further 
evaluation of the isospin dependence for the macroscopic component of fission barriers and search for the manifestation of collective effects in the de-excitation of nuclei. In this respect we note that the transitional region from spherical $\mathrm{Rn}$ nuclei to deformed ones corresponding to $N \leqslant 116$ [5] is accessible for study using very asymmetric combinations.
They allow one to deal with relatively high production cross sections and more reliable measurements of these values. The preliminary analysis of available $\mathrm{Rn}$ data shows their disagreement with each other [44] and requires the acquisition of new experimental information on the absolute production cross sections.
[1] S. Cohen, F. Plasil, and W. J. Swiatecki, Ann. Phys. (NY) 82, 557 (1974).

[2] M. Dahlinger, D. Vermeulen, and K.-H. Schmidt, Nucl. Phys. A376, 94 (1982).

[3] A. J. Sierk, Phys. Rev. C 33, 2039 (1986).

[4] W. D. Myers and W. J. Świątecki, Phys. Rev. C 60, 014606 (1999).

[5] P. Möller, J. R. Nix, W. D. Myers, and W. J. Swiatecki, At. Data Nucl. Data Tables 59, 185 (1995).

[6] W. Reisdorf, Z. Phys. A 300, 227 (1981); W. Reisdorf and M. Schädel, Z. Phys. A 343, 47 (1992).

[7] D. Vermeulen, H.-G. Clerc, C.-C. Sahm, K.-H. Schmidt, J. G. Keller, G. Münzenberg, and W. Reisdorf, Z. Phys. A 318, 157 (1984).

[8] C.-C. Sahm, H.-G. Clerc, K.-H. Schmidt, W. Reisdorf, P. Armbruster, F. P. Heßberger, J. G. Keller, G. Münzenberg, and D. Vermeulen, Nucl. Phys. A441, 316 (1985).

[9] A. V. Ignatyuk, K. K. Istekov, and G. N. Smirenkin, Yad. Fiz. 37, 831 (1983) [Sov. J. Nucl. Phys. 37, 495 (1983)].

[10] A. R. Junghans, M. de Jong, H.-G. Clerc, A. V. Ignatyuk, G. A. Kudyaev, and K.-H. Schmidt, Nucl. Phys. A629, 635 (1998).

[11] J. Tōke et al., Nucl. Phys. A440, 327 (1985).

[12] B. B. Back, Phys. Rev. C 31, 2104 (1985).

[13] W. Q. Shen et al., Phys. Rev. C 36, 115 (1987).

[14] J. G. Keller, B. B. Back, B. G. Glagola, D. Henderson, S. B. Kaufman, S. J. Sanders, R. H. Siemssen, F. Videbaek, B. D. Wilkins, and A. Worsham, Phys. Rev. C 36, 1364 (1987).

[15] A. Yu. Chizhov et al., Phys. Rev. C 67, 011603(R) (2003).

[16] G. N. Knyazheva et al., Phys. Rev. C 75, 064602 (2007).

[17] R. Rafiei, R. G. Thomas, D. J. Hinde, M. Dasgupta, C. R. Morton, L. R. Gasques, M. L. Brown, and M. D. Rodriguez, Phys. Rev. C 77, 024606 (2008).

[18] A. C. Berriman, D. J. Hinde, M. Dasgupta, C. R. Morton, R. D. Butt, and J. O. Newton, Nature (London) 413, 144 (2001); D. J. Hinde, A. C. Berriman, R. D. Butt, M. Dasgupta, I. I. Gontchar, C. R. Morton, A. Mukherjee, and J. O. Newton, J. Nucl. Radiochem. Sci. 3, 31 (2002).

[19] A. N. Andreyev et al., Phys. Rev. C 72, 014612 (2005).

[20] W. Reisdorf et al., Nucl. Phys. A438, 212 (1985).

[21] C. H. Dasso, S. Landowne, and A. Winther, Nucl. Phys. A407, 221 (1983).

[22] R. Bass, Phys. Rev. Lett. 39, 265 (1977); Lect. Notes Phys. 117, 281 (1980).

[23] G. Audi, A. H. Wapstra, and C. Thibault, Nucl. Phys. A729, 337 (2003).

[24] W. D. Myers and W. J. Swiatecki, Ark. Fys. 36, 343 (1967).

[25] R. N. Sagaidak et al., Phys. Rev. C 68, 014603 (2003).

[26] R. N. Sagaidak et al., in Proceedings of the 10th International Conference on Nuclear Reaction Mechanisms, Varenna, Italy,
2003, edited by E. Gadioli (Universitá degli Studi di Milano, Milan, 2003), p. 301; JINR Preprint E7-2003-149, Dubna, 2003.

[27] L. Corradi et al., Phys. Rev. C 71, 014609 (2005).

[28] R. N. Sagaidak et al., in Proceedings of the 11th International Conference on Nuclear Reaction Mechanisms, Varenna, Italy, 2006, edited by E. Gadioli (Universitá degli Studi di Milano, Milan, 2006), p. 279; JINR Preprint E7-2006-102, Dubna, 2006.

[29] J. van der Plicht, H. C. Britt, M. M. Fowler, Z. Fraenkel, A. Gavron, J. B. Wilhelmy, F. Plasil, T. C. Awes, and G. R. Young, Phys. Rev. C 28, 2022 (1983).

[30] R. J. Charity, J. R. Leigh, J. J. M. Bokhorst, A. Chatterjee, G. S. Foote, D. J. Hinde, J. O. Newton, S. Ogaza, and D. Ward, Nucl. Phys. A457, 441 (1986).

[31] R. Rafiei, R. G. Thomas, D. J. Hinde, M. Dasgupta, C. R. Morton, L. R. Gasques, M. L. Brown, and M. D. Rodriguez, Phys. Rev. C 77, 024606 (2008).

[32] C. R. Morton, A. C. Berriman, R. D. Butt, M. Dasgupta, A. Godley, D. J. Hinde, and J. O. Newton, Phys. Rev. C 62, 024607 (2000).

[33] D. J. Hinde, M. Dasgupta, J. R. Leigh, J. C. Mein, C. R. Morton, J. O. Newton, and H. Timmers, Phys. Rev. C 53, 1290 (1996).

[34] V. I. Zagrebaev et al., OM code of NRV, http://nrv.jinr.ru/nrv/.

[35] A. N. Andreyev, D. D. Bogdanov, A. V. Yeremin, A. P. Kabachenko, O. N. Malyshev, Yu.A. Muzychka, B. I. Pustylnik, R. N. Sagaidak, G. M. Ter-Akopian, and V. I. Chepigin, Yad. Fiz. 58, 791 (1995) [Phys. At. Nucl. 58, 730 (1995)]; Nucl. Phys. A583, 169 (1995).

[36] U. L. Businaro and S. Gallone, Nuovo Cimento 1, 1277 (1955).

[37] K. T. R. Davies and A. J. Sierk, Phys. Rev. C 31, 915 (1985).

[38] A. B. Quint et al., Z. Phys. A 346, 119 (1993); A. B. Quint, Ph.D. thesis, TH Darmstadt, GSI-89-22, GSI-89-23, 1989.

[39] A. V. Ignatyuk, G. N. Smirenkin, M. G. Itkis, S. I. Mulgin, and V. N. Okolovivh, Fiz. Elem. Chastits At. Yadra 16, 709 (1985) [Sov. J. Part. Nuclei 16, 307 (1985)].

[40] J. R. Nix, Nucl. Phys. A130, 241 (1969).

[41] F. Plasil and W. J. Swiatecki, see R. Vandenbosch and J. R. Huizenga, Nuclear Fission (Academic Press, New York, 1973), p. 246.

[42] A. V. Ignatyuk, K. K. Istekov, and G. N. Smirenkin, Yad. Fiz. 30, 1205 (1979) [Sov. J. Nucl. Phys. 30, 626 (1979)].

[43] L. G. Moretto, K. X. Jing, R. Gatti, G. J. Wozniak, and R. E Schmitt, Phys. Rev. Lett. 75, 4186 (1995).

[44] R. N. Sagaidak, submitted to Proceedings of the 2nd International Conference on Current Problems in Nuclear Physics and Atomic Energy (NPAE-Kyiv2008), Kyiv, Ukraine, 2008; JINR Preprint E7-2008-91, Dubna, 2008.

[45] S. Komarov, R. J. Charity, C. J. Chiara, W. Reviol, D. G. Sarantites, L. G. Sobotka, A. L. Caraley, M. P. Carpenter, and D. Seweryniak, Phys. Rev. C 75, 064611 (2007). 